\title{
Dependence of Supercavity Closure upon Flow Unsteadiness
}

Ashish Karn ${ }^{1,2}$, Roger E.A. Arndt ${ }^{1}$, Jiarong Hong ${ }^{1,2, *}$

1. Saint Anthony Falls Laboratory, $23^{\text {rd }}$ AVE SE, University of Minnesota, Minneapolis, MN, USA 55414.

2. Department of Mechanical Engineering, University of Minnesota, Minneapolis, MN, USA 55414.

* Email addresses of the corresponding author: jhong@umn.edu

\begin{abstract}
The present work reports some interesting experimental results for ventilated supercavitation in an unsteady flow. Our experiments have shown that incoming flow unsteadiness does not only affect supercavity shape but also leads to a change in supercavity closure, irrespective of the presence of a body inside the supercavity. Synchronized high-speed imaging and pressure measurements have ascertained the dependence of supercavity closure on instantaneous cavitation number under unsteady flow conditions. Further, control-volume analysis at the closure shows the intricate relation among the cavitation number, pressure difference occurring at the supercavity closure and the obtained closure mechanisms.
\end{abstract}

Keywords: Supercavitation, ventilation, supercavity closure, unsteady supercavitation

\section{Introduction}

Supercavitation is a special case of cavitation which can be employed to create a bubble of gas inside a liquid, large enough to encompass an object travelling through the liquid. The supercavitation phenomenon is of great practical interest owing to its advantages in the drag and noise reduction for high-speed underwater vehicles [1-3]. Such high-speed supercavitating 


\section{ETFS: Short Communication}

vehicles travel inside cavities which may be naturally or artificially generated [4]. A supercavity can be formed at lower speeds by injecting non-condensable gas, viz. air at the downstream of the cavitator, commonly referred to as artificial or ventilated supercavitation [5]. Generally, a ventilated supercavity can be characterized by three non-dimensional parameters: cavitation number, $\sigma=2\left(P_{\mathrm{O}}-P_{\mathrm{C}}\right) /\left(\rho_{\mathrm{w}} U^{2}\right)$; Froude number, $F r=U / \sqrt{g d_{\mathrm{C}}}$, and air entrainment coefficient, $C_{\mathrm{Q}}=\dot{Q} / U d_{\mathrm{C}}^{2}$, where $P_{\mathrm{O}}$ and $P_{\mathrm{C}}$ refers to the test-section pressure upstream of the cavitator and cavity pressure respectively, $\rho_{\mathrm{w}}, U$ and $g$ correspond to liquid density, the freestream velocity in the test-section and gravitational acceleration, respectively, $d_{\mathrm{C}}$ denotes the

cavitator diameter, and $\dot{Q}$ is the air ventilation rate. The study of supercavity closure, i.e. how a supercavity closes in the rear portion, is significant, since a majority of ventilated gas leaks from the closure region, and the ventilation demand to form and sustain a supercavity is dependent upon the closure mode of supercavity under different flow conditions [6-8].

Twin-vortex (TV) and re-entrant jet (RJ) are two major supercavity closure mechanisms reported in the published literature [9-13]. These studies have investigated the flow conditions that govern the formation and transition of different closure modes based on empirical formulations using dimensionless parameters. In most cases, different types of closure modes have been studied separately, and no universal physics-based model of supercavity closure and gas entrainment have been yet proposed [14]. Thus, the conditions of formation of a particular closure mode reported by different authors are not in agreement with each other [10,12-13].

Moreover, the behavior of supercavity closure is intricately related to the unsteadiness of the external flow conditions. In reality, for a supercavitating vehicle operating under the sea, the supercavity might encounter varying external flow conditions and unsteadiness particularly when the underwater vehicle moves close to the sea surface and encounters the effect of sea waves. 


\section{ETFS: Short Communication}

The unsteady flow induced by the movement below a wave train might result in fluctuations in angle of attack and can affect the supercavity. Till now, there have been very few studies on supercavitation in unsteady flows. The computational study by Semenenko on dynamic processes of supercavitation entailed an investigation into the change in supercavity shape upon introduction of an unsteady oncoming flow with a sinusoidal disturbance [15]. The recent study of Lee et al. provided further insight by examining the change in shape of a ventilated supercavity upon duplication of different sea-states [16]. Qi-tao et al. [17] investigated the case of a supercavitating vehicle pitching up and down in a ventilated supercavity to investigate the effect of impact of vehicle body and supercavity upon the evolution of pressure inside the cavity. Recent study by Nouri et al. [18] explored the dynamic characteristics of the cavity with the fluctuations caused by a change in supercavity parameters. However, the effect of flow unsteadiness upon supercavity closure has not yet been explored and investigated in detail.

The present work reports some experimental results for supercavitation in an unsteady flow, particularly focusing on the dependence of supercavity closure upon flow unsteadiness. The paper is structured as follows: Section 2 provides the details of the experimental methods. Subsequently, in section 3, high speed images of supercavity closures and the synchronized pressure measurements are presented for different unsteady states and a possible correlation between them is suggested. This is followed by a final conclusion in section 4 . 
ETFS: Short Communication

\section{Experimental Setup and Procedures}

The experiments are conducted at the Saint Anthony Falls Laboratory high-speed water tunnel. This water tunnel is a closed recirculating facility with a horizontal test-section of $1.20 \mathrm{~m}$ (Length) $\times 0.19 \mathrm{~m}($ Width $) \times 0.19 \mathrm{~m}($ Height $)$. The tunnel is specifically designed for cavitation and air ventilation studies and is capable of operating at velocities as high as $20 \mathrm{~m} / \mathrm{s}$. The unsteadiness in the flow is introduced by the means of a gust generator as in previous studies [19]. Figure 1a below shows a schematic of the experimental setup. The gust generator consists of two slender oscillating hydrofoils, which are placed upstream of the cavitator at a distance 180 $\mathrm{mm}$. These two hydrofoils oscillate in phase by the system's pivot arm to generate uniform gusts. This pivot arm is linked to a flywheel through a connecting arm, which extracts the periodic motion from the motor. The eccentricity flywheel can allow for gusts of varying amplitudes. Time-varying velocity amplitudes of periodic gust flows are measured with Laser Doppler Velocimetry (LDV) as described by Lee et al. [16]. The LDV measurements show that the frequency of the periodic gust flow in the test-section is equal to the oscillation frequency of the gust generator at each flow condition.

A cavitator with back surface facing the incoming flow, referred to as backward facing model, is employed in the experiment following the previous experiments by Kawakami and Arndt [13]. Figure $1 \mathrm{~b}$ illustrates the general placement of backward-facing model within the test section. As it shows, a hydrofoil strut is placed upstream of the cavitator to avoid the interaction between the formed cavity and the strut body leading to a free closure as reported by Logvinovich [20]. High speed videos of the supercavity are taken to ensure that the slender streamlined hydrofoil upstream does not produce significant disturbance on the cavity and only a faint wake signature could be observed. The model has special ventilation ports at the rear of the cavitator to create a 


\section{ETFS: Short Communication}

supercavity. The air flow rate is controlled by a mass flow controller and is kept steady during the experiments.

The hypodermic tube used for pressure measurement runs axially inside the ventilation line as shown in Figure 1c. Pressures in the test section and the supercavity are measured using two separate Validyne AP-10 pressure transducers. The pressure transducers are calibrated before each experiment and the pressure lines are purged with compressed air once before the pressure measurements are recorded at each condition. Uncertainty in the upstream pressure obtained using a pressure transducer is found to be less than 3\%. The Fast Fourier Transform and the subsequent plot of power spectral density of pressure data show that the dominant frequency exactly matches the oscillation frequency of the gust generator (not shown). The images and the videos of the supercavity closure are obtained with a $1 \mathrm{~K} \times 1 \mathrm{~K}$ pixel Photron APX-RS camera, which is capable of acquiring 3000 frames/s at full resolution. To ensure uniform backlighting in these videos and images, a light shaping diffuser is placed between the light source and the flow. The high speed imaging and the pressure measurements inside the cavity and test-section are synchronized using LABVIEW software. The experiments are conducted at a fixed $F r=20$ and $C_{\mathrm{Q}}=0.03$ with a cavitator diameter of $10 \mathrm{~mm}$, which corresponds to a minimal blockage of about $4.7 \%$. At the steady state, the closure observed is re-entrant jet, with a disturbance propagating in upstream direction as also observed in previous studies [21-23]. The gust amplitude is kept fixed at 10 degrees for all the experiments and the gust frequencies are varied between $1-10 \mathrm{~Hz}$. The pressure measurements and high speed videos are captured at a sampling rate of $1500 \mathrm{~Hz}$. 
(a)

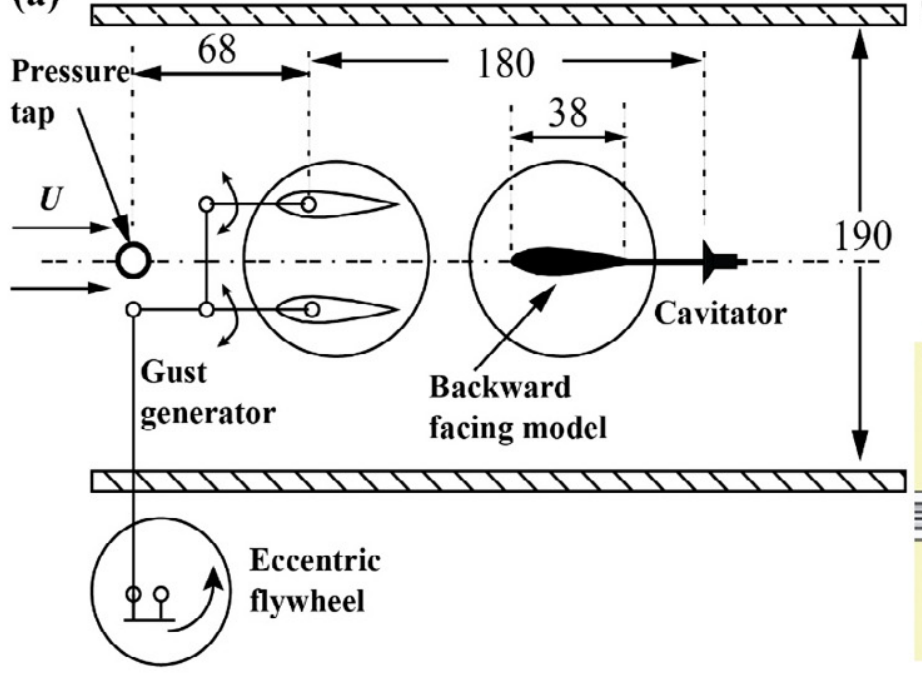

(b)
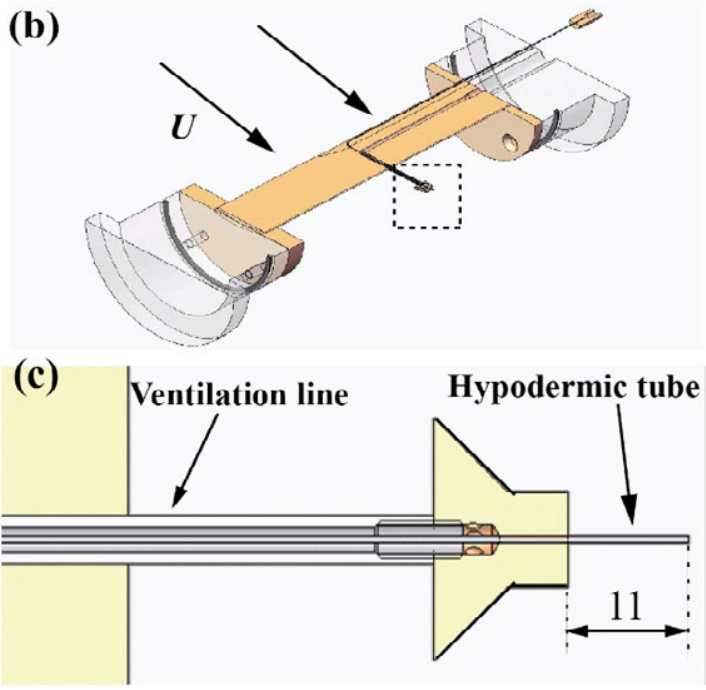

Fig. 1: (a) The experimental setup for studying unsteady ventilated supercavitation. (b) The schematic of the backward-facing cavitator model. (c) The close-up side view of the cavitator. All dimensions are in mm.

\section{Results and Discussion}

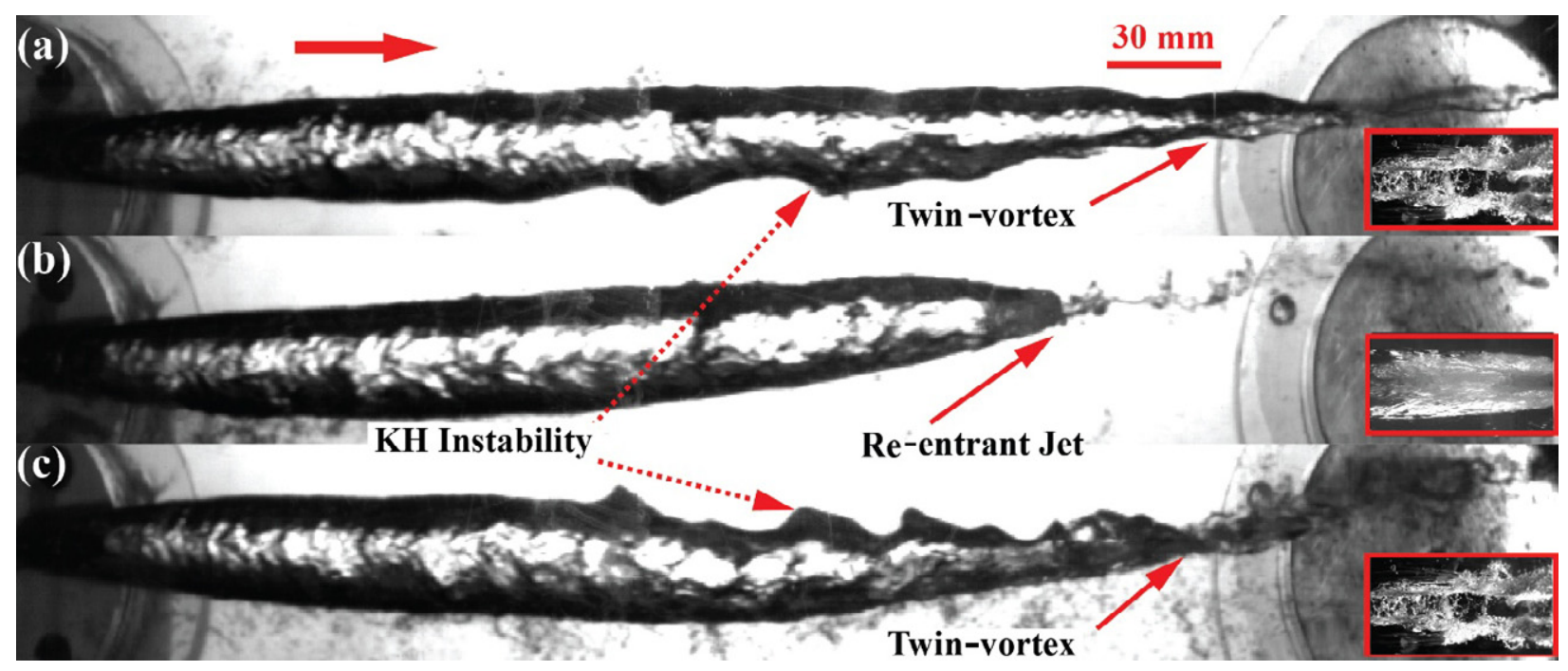

Fig. 2: The closure variations during a gust cycle with the backward-facing cavitator. The insets show the corresponding bottom views of these closure mechanisms. The arrow on the top left indicates the direction of the liquid flow. The scale bar applies to all three images.

Figure 2 shows the supercavity geometry at different time stamps during a gust cycle with the backward-facing cavitator. As the figure shows, the closure mode alternates between TV and RJ 


\section{ETFS: Short Communication}

closure in one gust cycle. In the TV closure, the supercavity closes in two counter-rotating hollow vortex tubes with opposite circulation whereas in the RJ closure, the cavity end is filled with foam which is periodically rejected by portions in the form of toroidal vortices. Although the side views of the closure cannot ascertain the presence of a TV closure, it is substantiated by the bottom views of the closures as shown in the insets of Figure 2a and Figure 2b. Careful observations of high speed videos indicate that $\mathrm{TV}$ is formed around the mean location of the oscillating hydrofoil (at the point of minimum blockage in the tunnel) whereas RJ occurs when the oscillating hydrofoils reach their extrema. Further, it is interesting to note the presence of Kelvin-Helmholtz $(\mathrm{KH})$ instability on the supercavity surface for a TV closure, while there is no such instability observed when RJ mode is present. The presence of this instability can be attributed to the unsteadiness in the incoming flow and the instantaneous rise in relative velocity between water and air [24]. This velocity rise is caused by the large tunnel blockage that is momentarily created at the amplitudes of the oscillating hydrofoils. Note that such closure transitions under unsteady flow conditions are not only limited to free closures, but they can also be observed when there is a body present inside the cavity as shown in Figure 3. In addition, for supercavities obtained with both backward and forward-facing cavitators, the expansion of each cross-section of the cavity is relative to the path of supercavity center, consistent with the principle of independent expansion given by Logvinovich [20]. Logvinovich's principle, which is considered to be an efficient tool for calculating the shape of long three-dimensional supercavities, further states that the supercavity shape also depends on velocity and pressure difference upstream and inside the cavity [25]. 


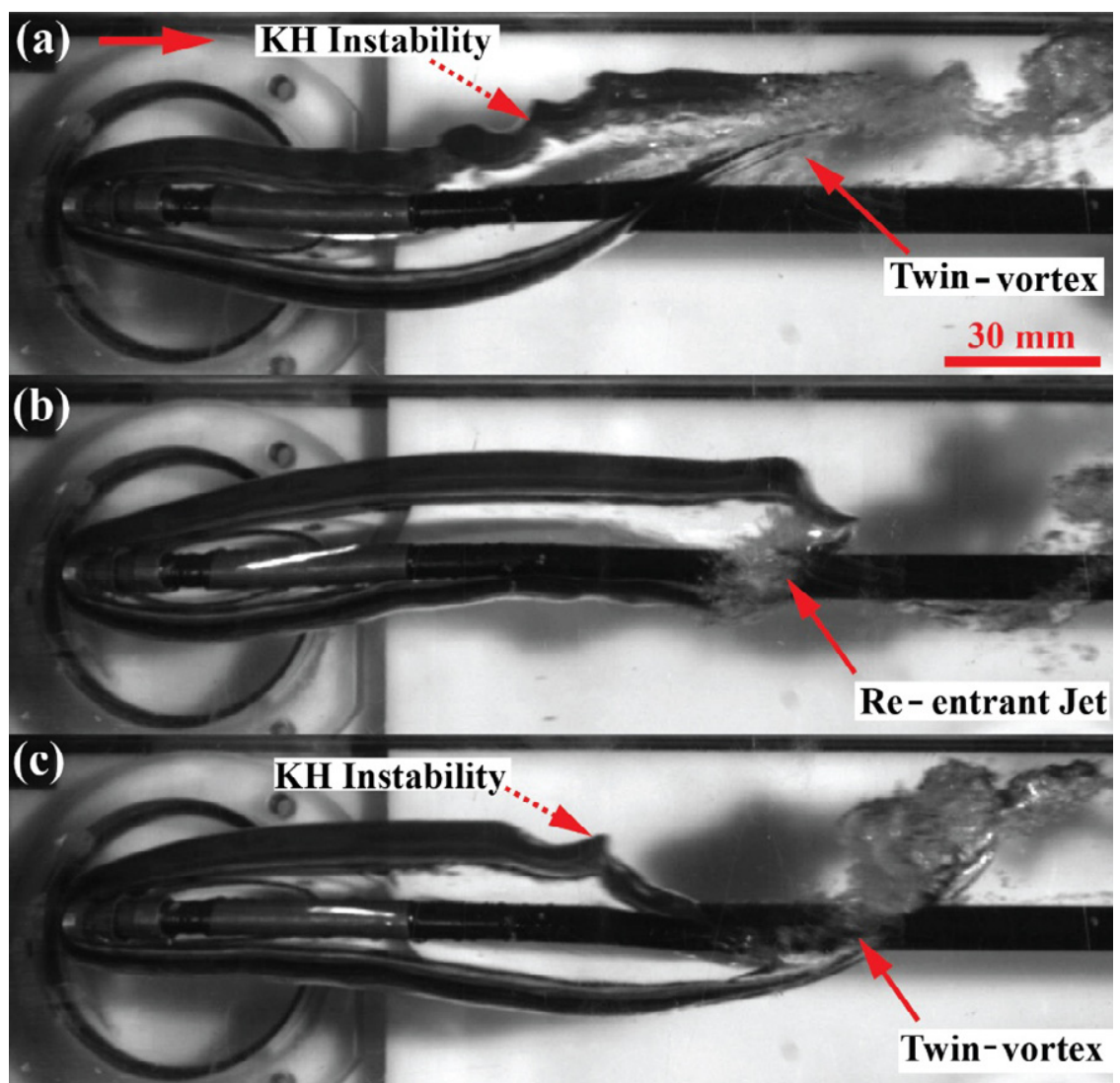

Fig. 3: The closure variations during a gust cycle with the forward-facing model. The arrow on the top left indicates the direction of the liquid flow. The scale bar applies to all three images.

To get an insight into the physical mechanisms that cause the change in cavity shape and closure, we conducted simultaneous pressure measurements both inside and outside the cavity in sync with the high-speed imaging of cavity behavior under different gust frequencies. To quantify the effect of unsteadiness, the pressure data are recorded at steady state $\left(F r=20, C_{Q}=\right.$ 0.03), under which RJ closure is present. The acquired values yield a non-dimensional testsection pressure $\left(\widetilde{P_{o}}=2 P_{\mathrm{O}} / \rho_{w} U^{2}\right)$ of 1.92 and non-dimensional cavity pressure $\left(\widetilde{P_{c}}=2 P_{\mathrm{C}} / \rho_{w} U^{2}\right)$ of 1.66 , respectively, corresponding to a cavitation number $\left(\sigma=\widetilde{P_{o}}-\widetilde{P_{c}}\right)$ of 0.26 . When unsteadiness is introduced at a low gust frequency of $0.71 \mathrm{~Hz}$ (Figure 4a), compared to the steady state values, $\widetilde{P_{O}}$ drops a little while $\widetilde{P_{c}}$ increases slightly, thus causing a decrement in $\sigma$. The flapping of the gust generator causes fluctuation of test-section pressure which in turn 


\section{ETFS: Short Communication}

leads to an oscillation in cavity pressure. However, as noted by Kuklinski et al. [24], pressure information in the liquid may propagate at different speeds than in the gas cavity, resulting in the variation of $\widetilde{P_{c}}$ that lags half cycle behind that of $\widetilde{P_{o}}$ in our experiments. This difference in phase of the test-section pressure and cavity pressure leads to the oscillating nature of $\sigma$. As the figure shows, $\sigma$ peaks at the maxima of $\widetilde{P_{o}}$ and minima of $\widetilde{P_{c}}$, varying between 0.12 and 0.22 . In the time series of $\sigma$, the RJ closure occurs at the crests $(\sigma=0.22)$ and TV mode coincides with the troughs $(\sigma=0.12)$. Compared to the steady state, the occurrence of TV closure effectively leads to a lower cavitation for the entire gust cycle. This observation is in agreement with the published reports of Semenenko [14] where the RJ closure is reported to have a higher value of $\sigma * F r$ as compared to TV closure. In our experiments, the parameter $\sigma * F r$ varies between 2.4 and 4.4 in one gust cycle as the closure modes alternate between TV and RJ respectively. In actuality, if the local increment in $F r$ due to the blockage caused by gust generator is considered, the actual value of $\sigma * F r$ are slightly greater. Thus, in all cases it is observed that both TV and $\mathrm{RJ}$ closure modes are present at $\sigma * F r>2.4$. However, this observed value of $\sigma * F r$ is in contradiction to the observations of Campbell and Hilborne [10] and many other researchers [11, 14] who proposed a critical value of unity for $\sigma * F r$ for TV-RJ closure transition.

This disagreement persists even when the experiments are carried out at a higher gust frequency of $7.10 \mathrm{~Hz}$. The value of $\sigma * F r$ varies in a narrower range of $3.2-3.9$ over the entire gust cycle, and the observed closure exhibit the characteristics of both twin vortex and re-entrant jet modes (referred to as TVRJ) as reported in Skidmore [8]. In addition, as shown in Figure 4b, both $\widetilde{P_{o}}$ and $\widetilde{P_{c}}$ move further away from their steady-state values. Although the trends in variation of $\widetilde{P_{o}}$ and $\sigma$ largely remain the same, the peak-to-peak variation of both $\widetilde{P_{o}}$ and 
$\sigma$ decreases considerably while the cavity pressure almost stays constant since it cannot match the high rate oscillations of unsteady ambient flow conditions.

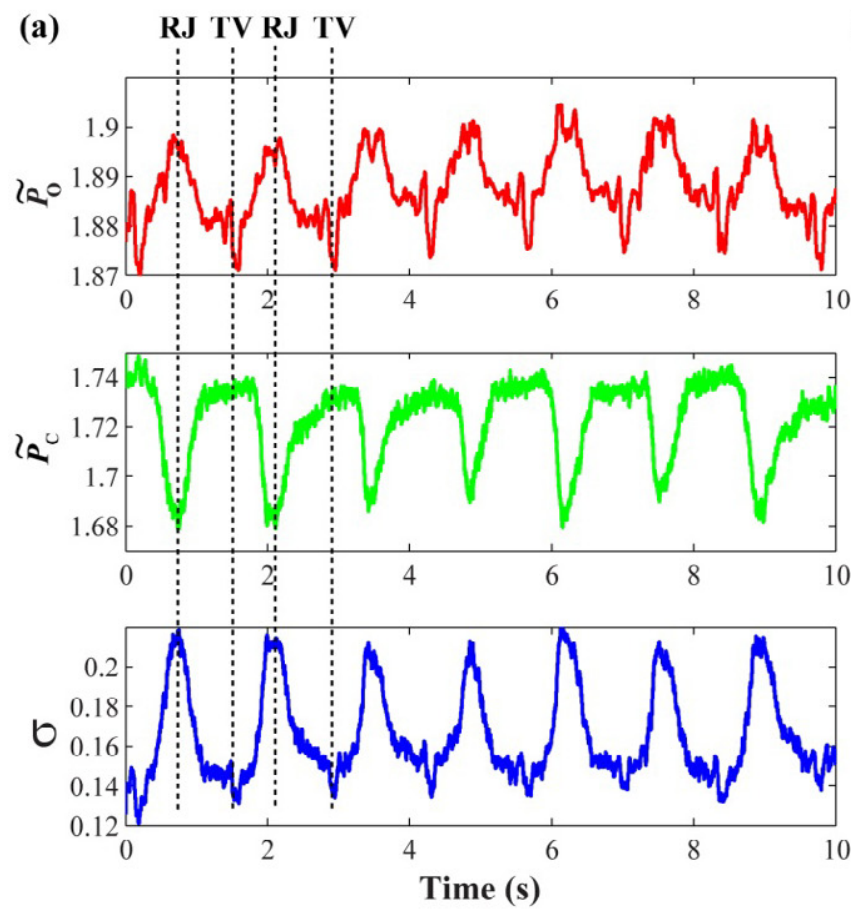

(b)
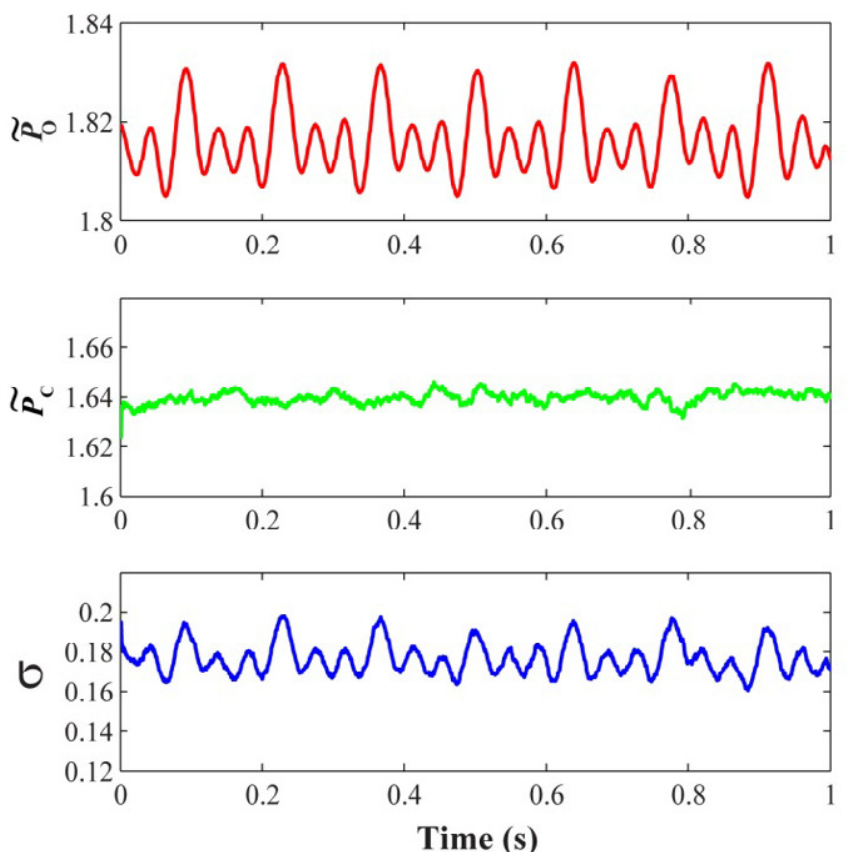

Fig. 4: The periodic variation of test section pressure, cavity pressure, and the cavitation number defined by their difference for the gust frequency at (a) $0.71 \mathrm{~Hz}$ and (b) $7.10 \mathrm{~Hz}$.

Finally, control volume (CV) analysis has been conducted at the RJ closure (CV-1) to provide some insight into the comparison between the pressure difference at the closure and the measurements of $\sigma$ from our experiments. As shown in Figure 5, $U$ indicates upcoming liquid velocity, $U_{\mathrm{RJ}}$ is the velocity of liquid rushing into the cavity, $U_{\mathrm{Cg}}$ and $U_{\mathrm{Og}}$ refer to the velocity of gas entering and exiting the $\mathrm{CV}-1$, respectively, $A_{\mathrm{C}}$ is the cross-sectional area of the $\mathrm{CV}-1$ on the cavity side whereas $A_{\mathrm{RJ}}$ refers to the effective area through which liquid enters into the cavity. $P_{\text {in }}$ denotes the pressures on the cavity side of CV-1 and $P_{\text {out }}$ represents the pressure outside CV-1. 
ETFS: Short Communication

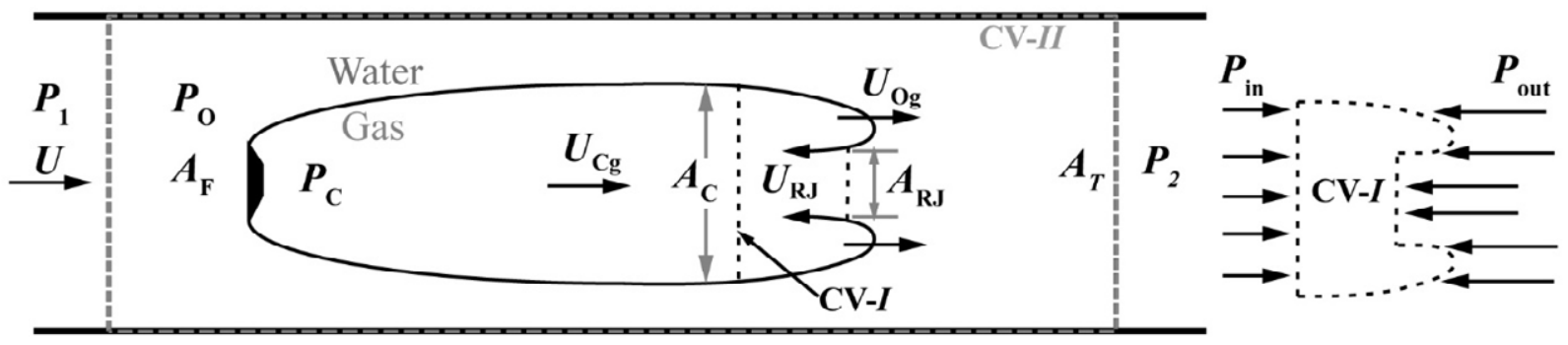

Fig. 5: Schematic showing the two control volumes: CV-1 is the control volume encompassing the cavity while CV-2 is a control volume at the RJ closure. The horizontal boundaries of CV-2 coincide with the test-section walls.

Based on the above definitions, the momentum equation for $\mathrm{CV}-1$ in the $x$-direction can be written as:

$$
\begin{gathered}
A_{C} P_{\text {in }}-A_{C} P_{\text {out }}=-A_{C} \rho_{g} U_{C g}{ }^{2}+\dot{M}_{\text {in }}-\dot{M}_{\text {out }} \\
\left(P_{\text {out }}-P_{\text {in }}\right) A_{C}=\left(A_{C} \rho_{g} U_{C g}{ }^{2}+A_{R J} \rho_{w} U_{R J}{ }^{2}\right)-A_{C} \rho_{g} U_{\text {og }}{ }^{2}
\end{gathered}
$$

An estimate of $P_{\text {out }}$ can be derived through momentum analysis of the control volume that completely encompasses the supercavity $(\mathrm{CV}-2)$. For $\mathrm{CV}-2$, the pressure acting on the left and the right surfaces are denoted by $P_{1}$ and $P_{2}$, respectively, $F_{\mathrm{D}}$ is the drag force on the cavitator, $A_{\mathrm{F}}$ and $A_{\mathrm{T}}$ are the frontal area of the cavitator and the cross-sectional area of the test-section, respectively. Then the relation between the pressures and drag force on the cavitator can be expressed as:

$$
P_{2} A_{\mathrm{T}}=P_{1} A_{\mathrm{T}}-F_{\mathrm{D}}
$$

Further, $P_{1} \approx P_{\mathrm{O}}$ and $P_{2} \approx P_{\text {out }}$

$$
P_{\mathrm{out}}=P_{\mathrm{O}}-\mathrm{F}_{\mathrm{D}} / \mathrm{A}_{\mathrm{T}}=P_{\mathrm{O}}-{ }_{2}^{1} * C_{\mathrm{D}} * A_{\mathrm{F}} * \rho_{w} * U^{2} / A_{\mathrm{T}}
$$

Considering that the momentum carried out by gas in comparison to liquid is negligible, and assuming $P_{\text {in }} \approx P_{\mathrm{C}}$ and negligible pressure drop across the cavity, i.e., $P_{\text {out }}=P_{\mathrm{O}}$, we can derive:

$$
\begin{gathered}
\left(P_{O}-P_{C}\right)-\frac{1}{2} * C_{D} * \mathrm{~A}_{F} * \rho_{w} * U^{2} / \mathrm{A}_{T} \approx \rho_{w}\left(A_{R J} / A_{C}\right) U_{R J}^{2} \\
2\left(P_{O}-P_{C}\right) / \rho_{w} U^{2} \approx 2\left(A_{R J} / A_{C}\right)\left(U_{R J} / U\right)^{2}+C_{D} * \mathrm{~A}_{F} / \mathrm{A}_{T}
\end{gathered}
$$




\section{ETFS: Short Communication}

Moreover, since the blockage in the test-section due to the cavitator is fairly minimal, the formation of the cavity does not lead to an appreciable increase in liquid velocity outside the cavity. Therefore,

$$
\sigma_{R J} \approx 2\left(A_{R J} / A_{C}\right)\left(U_{R J} / U\right)^{2}+C_{D} * \mathrm{~A}_{F} / \mathrm{A}_{T}
$$

Using high-speed videos of the RJ closure under the same $F r$ and $C_{\mathrm{Q}},\left(A_{R J} / A_{C}\right)$ and $\left(U_{R J} / U\right)$ is estimated to be about 0.5 , and a value of $C_{\mathrm{D}}=1.17$ is chosen for a disk in a turbulent flow [26]. Substituting these values in the above expression, we obtain: $\sigma_{R J} \approx 0.25$. Although the $\mathrm{CV}$ analysis is conducted for steady state, the calculated value of $\sigma_{R J}$ compares well with the maximal cavitation number (corresponding to RJ closure) for the unsteady state, particularly, at low gust frequency. This remarkable agreement indicates the connection between the fluctuation of cavitation number and the variation of flow patterns and pressure difference across the rear end of a supercavity. Specifically, we have shown that the variations in measured instantaneous cavitation number are coupled with the change in supercavity closure modes during a gust cycle. Thus, the transition among different supercavity closures are actually governed by the physical

processes that induce the pressure variation at the closure and flow pattern change inside supercavity, which cannot simply be demarcated by the value of $\sigma * F r$ as reported in previous studies. However, to substantiate this hypothesis would require systematic experimental investigations of closure modes under a wide range of flow conditions with a quantification of flow and pressure distributions within a supercavity.

\section{Conclusions}

In this short communication, some interesting insights into the transition of supercavity closure mechanisms in an unsteady flow are presented. Our experiments have shown that the incoming 


\section{ETFS: Short Communication}

flow unsteadiness simulated by periodic gusts, does not only affect supercavity shape but also leads to a change in supercavity closure. At a low gust frequency, it has been observed that under unsteady flow conditions, the supercavity closure alternates between TV and RJ modes at different instants for both backward and forward facing cavitators. Measurements at a higher frequency indicate a similar trend in the variation of pressures and the cavitation number, but the cyclical variation of closure modes becomes less distinct. As shown by simultaneous pressure measurements inside and outside the cavity and the synchronized high-speed imaging, the supercavity closure is a function of instantaneous cavitation number under unsteady flow conditions. Specifically, the presence of unsteadiness in the flow generates temporal fluctuations in the cavitation number, resulting in distinct closure modes. Further, control-volume analysis at the closure suggests that the cavitation number measurements in our experiments are intricately related to the pressure difference occurring at the closure, which could be the factual cause of occurrence of a variety of closure modes in ventilated supercavitation. These findings point out the significance of looking into the fundamental physics of ventilation supercavitation, which is beyond what the empirical relations (e.g. $\sigma * F r$ criterion) can offer.

\section{Abbreviations}

\begin{tabular}{|c|l|c|l|l|l|}
\hline$P_{\mathrm{O}}$ & Test-section pressure & $d_{\mathrm{C}}$ & Cavitator diameter & $C_{\mathrm{Q}}$ & Air entrainment coefficient \\
\hline$P_{\mathrm{C}}$ & Internal cavity pressure & $\dot{Q}$ & Air ventilation rate & $\mathrm{TV}$ & Twin-vortex \\
\hline$\rho_{\mathrm{w}}$ & Density of water & $\sigma$ & Cavitation number & $\mathrm{RJ}$ & Re-entrant Jet \\
\hline$U$ & Incoming liquid velocity & $\mathrm{Fr}$ & Froude number & $\mathrm{KH}$ & Kelvin-Helmholtz \\
\hline
\end{tabular}


ETFS: Short Communication

\section{Acknowledgments}

The authors gratefully acknowledge support by the Office of Naval Research (Program manager, Dr. Ronald Joslin) under Grant \# N000140910141. We also acknowledge the contribution of Mr. Ellison Kawakami for the closure images shown in Figure 3.

\section{References}

1. J.P. Franc, J.M. Michel, Fundamentals of Cavitation, Kluwer Academic Publishers, Dordrecht, 2004.

2. C.E. Brennen, Cavitation and Bubble Dynamics, Oxford University Press, Oxford, 1995.

3. I. Nesteruk, Supercavitation, Springer-Verlag, Berlin Heidelberg, 2012.

4. J.E. Dzielski, Longitudinal stability of a Supercavitating Vehicle, IEEE Journal of Oceanic Engineering 36(4) (2011) 562-570.

5. P.J.K. Cameron, P.H. Rogers, J.W. Doane, D.H. Gifford, An experiment for the study of free-flying supercavitating projectiles, J. Fluids Eng. 133 (2) (2011) 021303-1 - 021303-9.

6. D. R. Stinebring, M. L. Billet, J. W. Lindau, and R. F. Kunz, "Developed cavitation-cavity dynamics," Pennsylvania state university, University Park, Applied Research Lab, 2001.

7. J. W. Lindau and M. P. Kinzel, "Advancement and application of multi-phase CFD modelling to high speed supercavitating flows," DTIC document, The Pennsylvania State University Applied Research Laboratory (2013).

8. G. Skidmore, "The pulsation of ventilated supercavities," Master of Science thesis, Department of Aerospace Engineering, Pennsylvania State University (2013).

9. R. Cox and W. Clayden, "Air entrainment at the rear of a steady cavity," Proceedings of the Symposium on Cavitation in Hydrodynamics, London (1956).

10. I. J. Campbell and D. V. Hilborne, "Air entrainment behind artificially inflated cavity," Proceedings of the Second Symposium on Naval Hydrodynamics, Washington (1958).

11. V. N. Buyvol, "Slender cavities in flows with perturbations" (in Russian). Nauvoka Dunka Ed. Kiev, Ukarine (1980).

12. J. Spurk, "On the gas loss from ventilated supercavities," Acta Mechanica, 155 (2002) 125135. 
ETFS: Short Communication

13. E. Kawakami and R. E. A. Arndt, "Investigation of the behavior of ventilated supercavities,” J. Fluids Eng. 133 (9) (2011) 091305-1 - 091305-11.

14. V. N. Semenenko, "Artificial Supercavitation: Physics and Calculation," Ukrainian academy of sciences, Kiev Inst. of hydromechanics (2001).

15. V. N. Semenenko, "Dynamic processes of supercavitation and computer simulation," Ukrainian Academy of sciences, Kiev Inst. of hydromechanics (2001).

16. S. J. Lee, E. Kawakami, and R.E.A. Arndt, "Investigation of the Behavior of Ventilated Supercavities in a Periodic Gust Flow,” J. Fluids Eng. 135 (8) (2013) 081301-1 - 081301-13.

17. L. Qi-tao, X. Lei-ping, H. You-sheng, Experimental study of ventilated supercavities with a dynamic pitch model, J. Hydrodyn. Ser. B 20(4) (2008) 456-460.

18. N.M. Nouri, R. Madoliat, Y. Jahangardy, M. Abdolahi, A study on the effects of fluctuations of the supercavity parameters, Exp. Thermal Fluid Sci. 60 (2015) 188-200.

19. J. Kopriva, R.E.A Arndt, and E. L. Amromin, "Improvement of hydrofoil performance by partial ventilated cavitation in steady flow and periodic gusts," J. Fluids Eng. 130 (3) (2008) 031301-1 - 031301-7.

20. G. V. Logvinovich, "Hydrodynamics of free-boundary flows," Halsted Press, 1973.

21. X. Wu, G.L. Chahine, Characterization of the content of the cavity behind a high-speed supercavitating body, J. Fluids Eng. 129(2) (2007) 136-145.

22. I. Rashidi, Ma. Pasandideh-Fard, Mo. Pasandideh-Fard, N.M.Nouri, Numerical and Experimental study of a ventilated supercavitating vehicle, J. Fluids Eng. 136 (2014) 101301-1 - 101301-7.

23. B. Stutz, J.L. Reboud, Experiments on unsteady cavitation, Exp. Fluids 22 (1997) 191-198.

24. R. Kuklinski, J. Castano, and C. Henoch, "Experimental study of ventilated cavities on dynamic test model," CAV 2001: Fourth International Symposium on Cavitation. Naval Undersea Weapons Center, Newport, RI (2001).

25. I. Nesteruk, Shape of slender axisymmetric ventilated supercavities, J. Comput. Eng. 2014 (2014).

26. R. W. Fox, Alan T. McDonald, and Philip J. Pritchard. "Introduction to fluid mechanics". Vol. 5., John Wiley \& Sons, New York, 1998. 